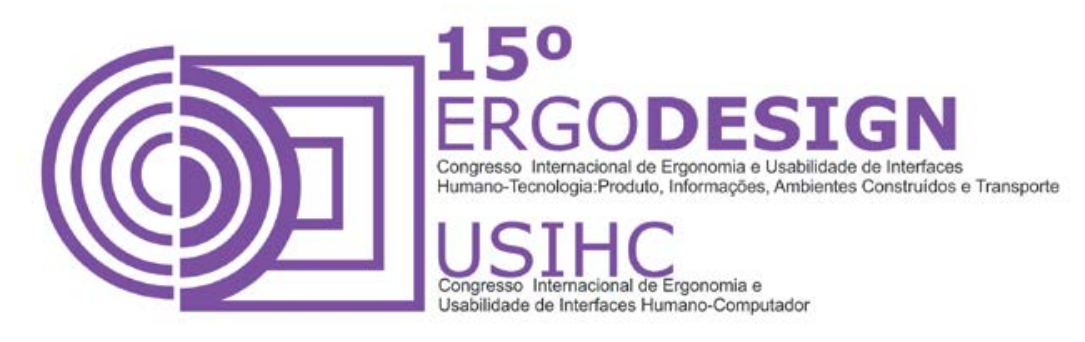

\title{
DESIGN RESPONSIVO E ACESSIBILIDADE PARA DISPOSITIVOS MÓVEIS: UMA REVISÃO SISTEMÁTICA DE LITERATURA
}

\author{
RESPONSIVE DESIGN AND ACCESSIBILITY FOR MOBILE DEVICES: A \\ SYSTEMATIC REVIEW OF LITERATURE \\ RIBAS, Armando (1); \\ VANZIN, Tarcisio (2); \\ ULBRITCHT, Vania (3); \\ (1) Universidade Federal de Santa Catarina, Mestre \\ e-mail: mandorgr@gmail.com \\ (2) Universidade Federal de Santa Catarina, Doutor \\ e-mail: tvanzin@gmail.com \\ (3) Universidade Federal de Santa Catarina, Doutora \\ e-mail: vrulbricht@gmail.com
}

\begin{abstract}
RESUMO
Este artigo responderá a seguinte questão de pesquisa: Há diretrizes e/ou critérios para criar páginas, sistemas WEB com acessibilidade utilizando a técnica de Design Responsivo? Para tanto foi realizada, em 2014, uma Revisão Sistemática de Literatura (RSL), considerada como uma conexão, ou agrupamento relacionando várias pesquisas para responder a uma pergunta bem definida. Como resultado mereceram destaque os autores Ethan Marcotte, precursor do Design responsivo, e Jakob Nielsen, considerado o pai da usabilidade.
\end{abstract}

Palavras-chave: Design Responsivo, Acessibilidade, Revisão Sistemática, Dispositivos Moveis. 


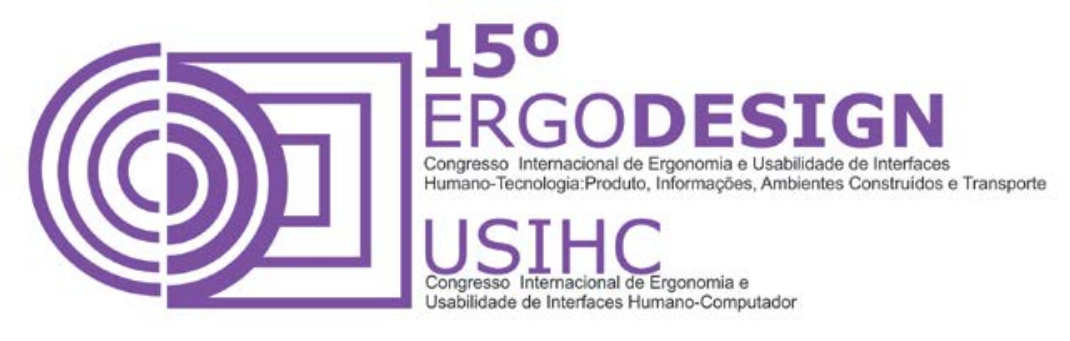

ABSTRACT

This article responderá a SEGUINTE Questão de Pesquisa: Ha Diretrizes e / ou criterios Pará Páginas Criar, Sistemas Web com Acessibilidade utilizando a Técnica de projeto Responsivo? Para tanto foi realizada, em 2014, Uma Revisão Sistemática de Literatura (RSL), Considerada Como Uma Conexão, or agrupamento relacionando VÁRIAS Pesquisas Pará responder uma pergunta Uma Bem Definida. Como resultado mereceram destaque OS Autores Ethan Marcotte, precursor do Design responsivo, e Jakob Nielsen, considerado o pai da Usabilidade.

Keywords: Responsive Design, Accessibility, Systematic Review, Mobile Devices;

\section{INTRODUÇÃO}

Os primeiros sites na época da WEB 1.0 possuíam muitos textos, poucas imagens e utilizavam somente tecnologia HTML. Não utilizavam sons e animações nem quaisquer outros sistemas multimidiáticos para deixar o ambiente WEB atrativo ao usuário e pouco se falava de acessibilidade. Os usuários, nessa época, realizavam leituras e mantinham contatos através dos chat e e-mail, ou seja, o usuário era "passivo" não interagia com o site (SILVA, 2009, p. 27).

Com a WEB 2.0 o usuário tornou-se "ativo", pois além de serem leitores, também são colaboradores (escritores), participando efetivamente para a construção do conhecimento. Desta forma, a WEB 2.0 não está diretamente ligada somente à tecnologia, mas na forma das pessoas agirem, como também, nos serviços prestados pelas instituições (MARINHO, 2006). Torres (2009) corrobora com Marinho, afirmando que a WEB 2.0 é dinâmica, pois há participação do usuário como produtor e consumidor de informações, criando assim, uma grande quantidade de informações, isto faz que os usuários, possam ter sobrecarga cognitiva .

Com o surgimento da WEB 3.0, também conhecida como WEB Semântica, a sobrecarga cognitiva dos usuários, foi minimizada. Pois esta WEB tem como proposito permitir que agentes de softwares interpretem os conteúdos da internet auxiliando os usuários a localizar as informações solicitadas em um menor tempo (W3C, 2013). Isso foi possível graças às novas tecnologias e metodologias que auxiliaram a transformar a WEB com característica global, isto é, reutilizar e compartilhar informações de diversos usuários. Mas para que isso ocorresse, foi necessário utilizar metodologias e tecnologias como: Tableless, CSS, CSS3, XHTM, XML (Extensible Markup Language), RDF(Resource Description Framework), HTML5, e Java-Script. Estas Tecnologias vieram com o intuito de melhorar a interface de sites e sua acessibilidade (W3C, 2013).

Gonçalves (2007) acrescenta, que a WEB semântica não é uma revolução da WEB 2.0, mas uma ampliação desta, através de métodos e técnicas definidas pela W3C, que tem como intuito criar um meio global para troca de informações, as quais devem ser entendidas por todos os seres humanos, como também, pelos computadores.

Além da evolução da WEB, há também a evolução dos dispositivos para acessar a internet, pois atualmente a tecnologia está em um constante estado de modificação e atualização. 


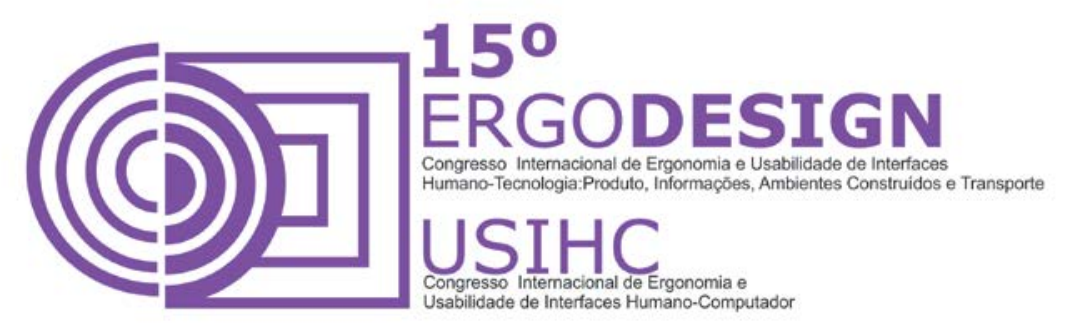

Percebe-se essa evolução e transformação, pelo crescente número de celulares e tablets com acesso a internet. No Brasil, os celulares ganham destaque, pois "[...] são oferecidos, com uma variada gama de tecnologia embarcada, em função da diversidade da sua utilização" (IBGE, 2013, p. 299).

Segundo o International Data Corporation (IDC, 2014), além dos celulares, os tablets também estão ganhando evidência. Pois há uma forte tendência de crescimento dessas duas tecnologias em comparação aos notebooks e desktops. No entanto, isso não se trata da substituição das tecnologias, mas de uma convergência das mesmas, havendo uma mudança social e cultural na utilização dessas tecnologias pelas pessoas e pelas empresas.

Sendo assim, é possível verificar que a cada dia há um aumento significativo de novos dispositivos e usuários com competências e conhecimentos distintos na utilização da WEB. Isto faz com que a usabilidade e acessibilidade sejam fundamentais para uma boa intervenção destes usuários nos sites, sistemas ou mídias para WEB.

Atualmente, autores como Nielsen(2011), Bastien \& Scapin (1993) e Cybes (2002) tratam do assunto acessibilidade e usabilidade WEB para notebooks e desktops, não abordando dos dispositivos móveis e das tecnologias e/ou metodologias para a criação de sistemas Web com características de Design Responsivo.

A W3C (2013) afirma que não há diretrizes de acessibilidade específica para dispositivos móveis, partindo do pressuposto que uma página WEB deve ser acessada por todos, independentemente do dispositivo de acesso. Mas como fazer isso, se nas recomendações da W3C o foco da acessibilidade é sempre para desktop?

Desta necessidade surgiram os sites criados com metodologia de Design Responsivo. Pois esta técnica faz o sistema Web se adaptar, modificando menus, mídias, texto e conteúdo, conforme o tamanho da tela e do dispositivo. Desta forma faz necessário criar métodos de desenvolvimento de acessibilidade para diversos dispositivos, pois cada dispositivo possui características, tamanhos e hardwares diferentes.

\section{REVISÃO SISTEMÁTICA}

A revisão sistemática de literatura (RSL) é uma técnica de pesquisa realizada sobre um determinado tema, utilizando métodos explícitos e metódicos para identificar e selecionar os arquivos mais pertinentes a pesquisa, como também, avaliá-los criticamente(POCINHO, 2008).

Galvão, Sawada e Trevizan (2004) complementam esta definição afirmando que uma RSL é como uma conexão, um agrupamento relacionando várias pesquisas com uma explícita questão. É considerada como uma forma de resumo das informações disponíveis sobre um problema específico, que é analisado e estudado por meio de métodos científicos escolhidos pelo autor (LIMA, 2003).

Atualmente a RSL está cada vez mais disseminada na pesquisa científica de várias áreas do conhecimento pelo enorme e crescente número de informações das diversas áreas do saber. Esse método auxilia a organizar e melhorar a pesquisa bibliográfica, integrando informações de 


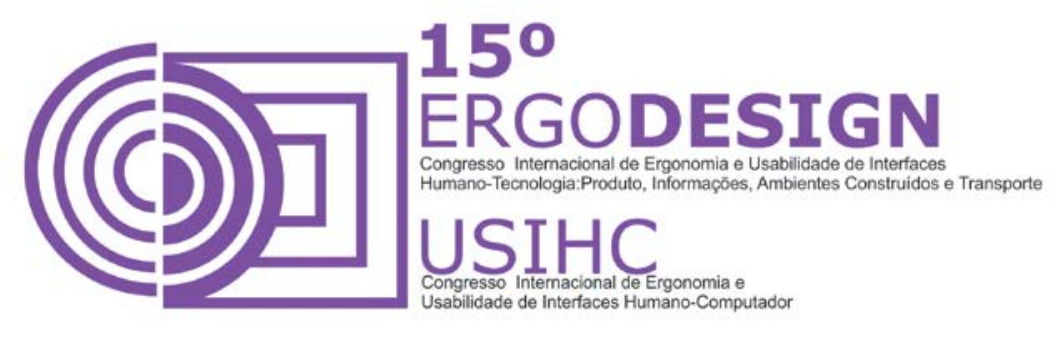

um estudo feito separadamente sobre um tema específico, podendo apresentar resultados que se coincidem, e/ou se divergem. (LINDE, 2003).

O método da pesquisa sistemática segundo Galvão (2004) possui alguns princípios gerais como:

- A exaustão na busca dos estudos analisados;

- A seleção justificada dos estudos por critérios de inclusão e exclusão;

- A avaliação da qualidade metodológica;

- A quantificação do efeito dos tratamentos por meio de técnicas estatísticas.

Obregon (2011) e, Linde (2003) afirmam que esse tipo de revisão é criado para responder uma ou muitas perguntas específicas de um tema explícito, e possui técnicas específicas para localizar, selecionar e avaliar criticamente o objeto estudado.

Neste trabalho utilizar-se-á a RSL qualitativa pois busca-se explicar o porquê dos eventos, não se importando pela quantificação dos valores, uma vez que os dados analisados são não métricos e se valem de diferentes abordagens.

\subsection{Seleção dos Bancos de Artigos}

Segundo Collaboration (2013) para se fazer uma revisão sistemática é necessário, primeiramente, definir uma pergunta de pesquisa, que neste trabalho foi esboçada como: Há diretrizes e/ou critérios para criar páginas, sistemas WEB com acessibilidade utilizando a técnica de Design Responsivo?

Com a pergunta definida, definiu-se as bases de dados a serem consultadas, que nesse caso serão: a Scopus, escolhida por ser considerada a maior do mundo, possuindo mais de 46 milhões de registros entre periódicos, revistas de livre acesso, anais de conferencias, dentre outros (SCOPUS, 2012). A segunda base utilizada foi a da Biblioteca Universitária (BU) da Universidade Federal de Santa Catarina (UFSC), escolhida por ser uma base local e possuir mais de 23 milhões de documentos entre livros, teses, dissertações. A terceira base trabalhada foi o portal da CAPES, pelo fato de ser uma base considerada sólida e confiável. Por ultimo foi escolhida a base da dados da Scielo, por ser uma base internacional e nacional e por apresentar arquivos de qualidade.

A pesquisa se restringiu a trabalhos completos, gratuitos e publicados nos últimos cinco anos que possuem em seu título uma das palavras-chaves, definidas para essa pesquisa segunda a tabela 2.

\subsection{Seleção das Palavras-Chave e Resultados}




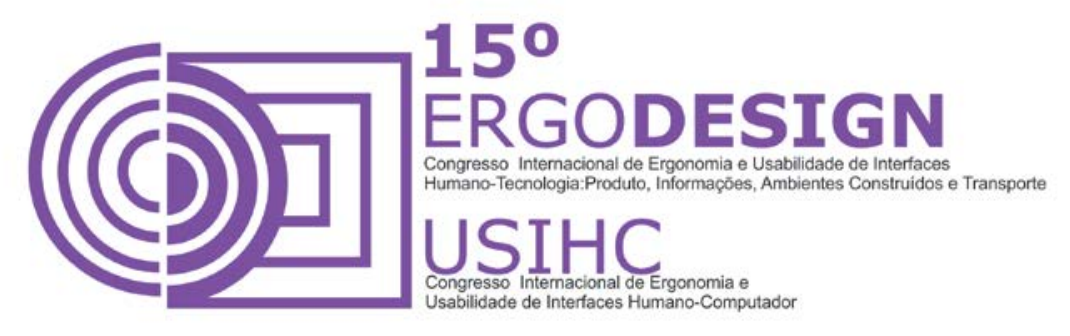

Com base no levantamento efetuado, foram localizados cinco palavras-chave, que foram ordenadas pela quantidade de vezes que apareceram na definição do problema, deste trabalho. A tabela 1 apresenta a lista com as palavras-chave incluindo também a língua inglesa, principal língua utilizada em pesquisa internacional.

Tabela 1 - Palavras-chaves mais identificadas e tradução para o inglês

\begin{tabular}{|c|c|}
\hline Português & Inglês \\
\hline Design responsivo & responsive Design \\
\hline Usabilidade & Usability \\
\hline Acessibilidade & accessibility \\
\hline Dispositivos moveis & mobile Devices \\
\hline Smartphone & Smartphone \\
\hline
\end{tabular}

Fonte: do autor, 2014

Com estas palavras, formou-se quatro combinações, Design responsivo e Acessibilidade; Design responsivo e Usabilidade; Design responsivo e dispositivos moveis e Design responsivo e smartphone.

Os resultados das pesquisas utilizando, as combinações descritas estão explicitadas na tabela 2.

Tabela 2 - Quantidades de artigos extraídas pelas combinação das palavras-chaves dos e banco dados

\begin{tabular}{|c|c|c|c|c|}
\hline $\begin{array}{c}\text { Banco de } \\
\text { Artigos }\end{array}$ & \multicolumn{5}{|c|}{$\begin{array}{c}\text { Total } \\
\text { e Acessibilidade }\end{array}$} & $\begin{array}{c}\text { Design responsivo } \\
\text { e Usabilidade }\end{array}$ & $\begin{array}{c}\text { Design responsivo } \\
\text { e dispositivos } \\
\text { moveis }\end{array}$ & Design responsivo e smartphone \\
\hline Capes & 3 & 4 & 23 & 7 \\
\hline Scopus & 3 & 2 & 9 & 2 \\
\hline $\begin{array}{c}\text { Biblioteca } \\
\text { UFSC }\end{array}$ & 0 & 0 & 0 & 0 \\
\hline
\end{tabular}




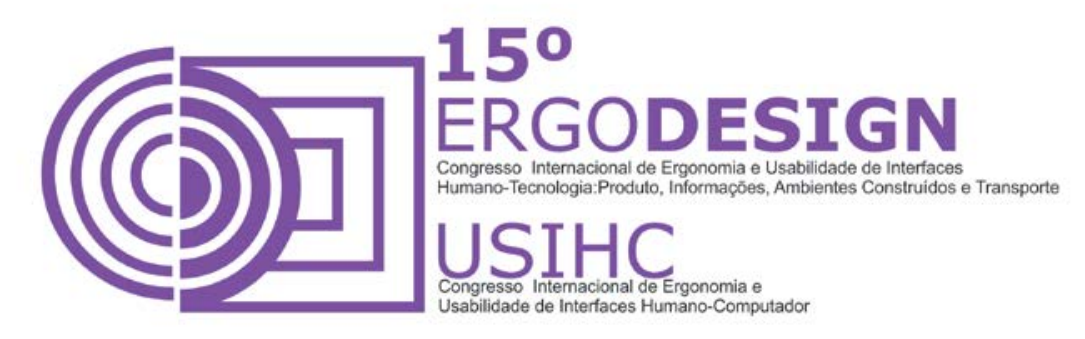

\begin{tabular}{|c|c|c|c|c|}
\hline Scielo & 0 & 0 & 0 & 0 \\
\hline Sub total & 6 & 6 & 32 & 6 \\
\hline TOTAL & \multicolumn{2}{|c|}{50} \\
\hline
\end{tabular}

Fonte: do autor, 2015

Foram utilizados os arquivos completos e gratuitos e que contem em seu título pelo menos uma das palavras-chave. Estes trabalhos estão colocados na tabela 3. 


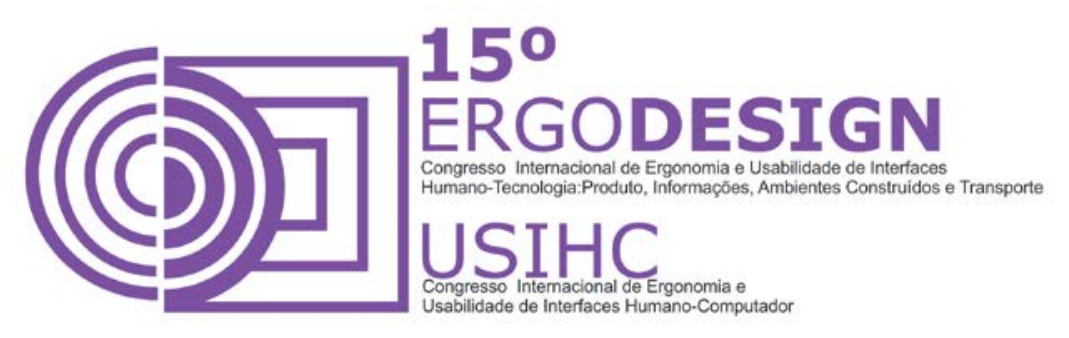

Tabela 3 - Quantidades de artigos extraídas que contenhas uma das palavras-chaves no título do trabalho

\begin{tabular}{|c|c|c|c|c|}
\hline $\begin{array}{c}\text { Banco de } \\
\text { Artigos }\end{array}$ & \multicolumn{5}{|c|}{$\begin{array}{c}\text { Total } \\
\text { e Acessibilidade }\end{array}$} & $\begin{array}{c}\text { Design responsivo } \\
\text { e Usabilidade }\end{array}$ & $\begin{array}{c}\text { Design responsivo } \\
\text { e dispositivos } \\
\text { moveis }\end{array}$ & Design responsivo e smartphone \\
\hline Capes & 3 & 4 & 5 & 3 \\
\hline Scopus & 1 & 1 & 6 & 1 \\
\hline $\begin{array}{c}\text { Biblioteca } \\
\text { UFSC }\end{array}$ & 0 & 0 & 0 & 0 \\
\hline Scielo & 0 & 0 & 0 & 0 \\
\hline Sub total & 4 & 5 & 11 & 4 \\
\hline
\end{tabular}

Do total de 24 artigos encontrados nas diferentes bases de dados, tabela 4 , dois foram eliminados por não possuírem uma das palavras chaves no título, sete por não serem gratuitos, restando15 artigos. Na leitura do resumo, cinco foram eliminados, pois não tinham pertinência a este trabalho e um (1) por estar duplicado (tabela 4 ).

Os trabalhos restantes, tabela 4, foram utilizados para o embasamento teórico deste trabalho.

Tabela 4 - Artigos e autores mais pertinentes elou citados

\begin{tabular}{|l|l|l|l|}
\hline $\begin{array}{c}\text { Base de } \\
\text { dados }\end{array}$ & \multicolumn{1}{|c|}{ Título artigos } & Autores & $\begin{array}{c}\text { Número de } \\
\text { aparições }\end{array}$ \\
\hline CAPES & $\begin{array}{l}\text { 5th International Conference on Software Development and } \\
\text { Technologies for Enhancing Accessibility and Fighting Info- } \\
\text { exclusion, DSAI 2013 }\end{array}$ & Wald & 3 \\
\cline { 2 - 4 } & $\begin{array}{l}\text { Becoming Responsive: Mobile Web Gets a Fresh Look as News } \\
\text { Organizations Turn to Responsive Design }\end{array}$ & Marcotte & 1 \\
\cline { 2 - 4 } & $\begin{array}{l}\text { That Was Then, This Is Now: Replacing the Mobile-Optimized } \\
\text { Site with Responsive Design }\end{array}$ & Nielsen & 3 \\
\cline { 2 - 4 } & Law Firm Websites for the iPad Generation & Algeri & 1 \\
\hline
\end{tabular}




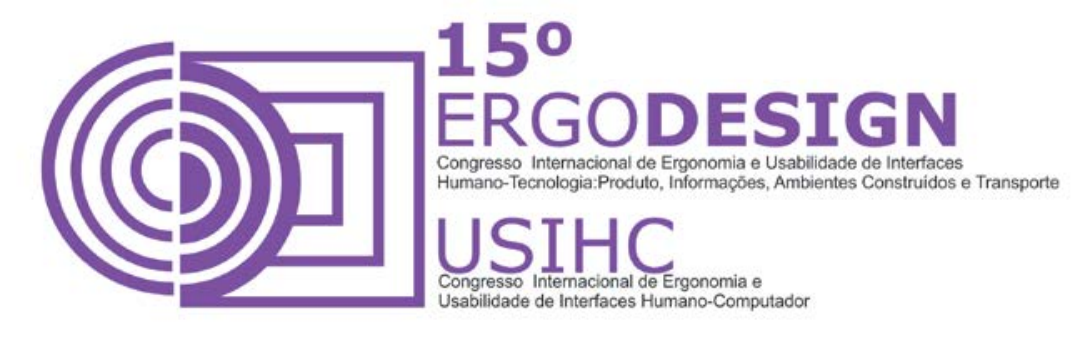

\begin{tabular}{|c|c|c|c|}
\hline \multirow[t]{9}{*}{ SCOPUS } & \multirow[t]{2}{*}{$\begin{array}{l}\text { Automatic Generation of Social Media Snippets for Mobile } \\
\text { Browsing }\end{array}$} & Yang & 2 \\
\hline & & Zhang & 4 \\
\hline & \multirow[t]{3}{*}{ Mobile Access to Information in the Agrarian Sector } & Yang & 1 \\
\hline & & Jarolímek & 3 \\
\hline & & Heitkötter & 2 \\
\hline & $\begin{array}{l}\text { Accessibility of the Regional Information Based on the Regional } \\
\text { Information Structure Framework }\end{array}$ & Jarolímek & 1 \\
\hline & \multirow[t]{2}{*}{ H1DS: A new web-based data access system } & Django & 3 \\
\hline & & Python & 2 \\
\hline & $\begin{array}{l}\text { That Was Then, This Is Now: Replacing the Mobile-Optimized } \\
\text { Site with Responsive Design }\end{array}$ & Marcotte & 1 \\
\hline \multicolumn{4}{|l|}{ CIELO } \\
\hline UFSC & & & \\
\hline
\end{tabular}

Nos dez (10) trabalhos restantes, foram localizadas 102 bibliografias, destas 40 são referencias de sites (sem autor definido), 76 possuem distintas autorias, e em 26 bibliografias os autores se repetem. A tabela 5 apresenta os autores mais citados e o número de vezes que aparecem, como também, os mais relavantes.

Destas bibliografia se destacam os autores Ethan Marcotte, precursor do Design responsivo, e Jakob Nielsen, considerado o pai da usabilidade.

\subsection{Bibliometria com Autores}

Aperfeiçoando a pesquisa foi necessário extrair dos trabalhos as referencias bibliográficas e relacionar os autores. Os nomes dos autores foram listados e ordenados alfabeticamente, sendo eliminado as referenciais duplicadas. Para finalizar, foi colocado em ordem os autores por quantidade de aparições. Nesse trabalho não há diferença entre autor e co autor.

Os autores como Zhang esta no referencial bibliográfico, mas não é citado em nenhum momento nos artigos apresentados; Jarolímek e Heitkötter são citados duas vezes no mesmo artigo, o primeiro aborda sobre acessibilidade de páginas/aplicativos WEB para agricultura em 


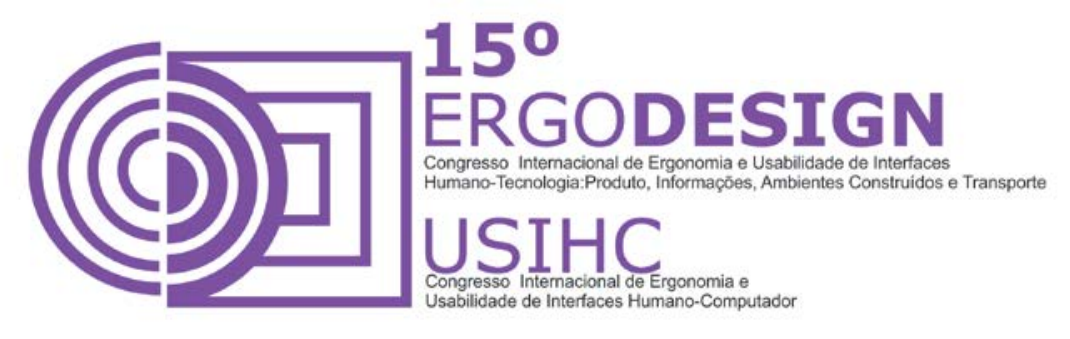

dispositivos moveis. O segundo autor foca suas citações e afirmações sobre programação WEB sempre comparando o desenvolvimento multi-plataforma para aplicações móveis.

A citação de Django é um site que trata sobre framework onde utilizou a linguagem de programação Python. O Wald e Python são sites que abordam documentos relativos a linguagem Python e a frameworks para promover a portabilidade de aplicações WEB através de uma variedade de servidores.

\section{CONCLUSÃO}

Este trabalho descreve o processo de Revisão Sistemática de Literatura para a identificação de conceitos que possam ser usados para responder a questão de há diretrizes e/ou critérios para criar, sistemas WEB com acessibilidade utilizando a técnica de Design Responsivo? Para tal foram selecionados quatro bancos de dados, cinco palavras-chave foram usadas e combinadas a busca inicial apontou 50 artigos que, após seguidas constrições, levaram a identificar 24 artigos e, após a leitura dos artigos, dez foram extraídos e foram usados na revisão.

$\mathrm{Na}$ avaliação crítica dos estudos, o banco de dados que mais teve artigos selecionados foram das bases Scopus e Capes. Nos bancos de dados Biblioteca Central da UFSC e Scielo, mesmo em português, nenhum trabalho foi apresentado.

Dos dez artigos extraídos, os autores foram listados, ordenados, identificando-se um total de 102 autores diferentes, destes 24 se repetem. Mas os que são relevantes a essa pergunta de pesquisa são somente dois o Ethan Marcotte, e Jakob Nielsen.

Com base nessa RSL, não foi encontrada nenhuma diretriz de acessibilidade para ser empregada na técnica de design responsivo, foram somente localizados artigos que tratam de assuntos como desenvolvimento WEB, Framework, trabalhos relacionados a importância do dispositivo móvel nas empresas.

\section{REFERÊNCIAS BIBLIOGRÁFICAS}

GALVÃO, C M; SAWADA, N. O.; TREVIZAN, M A. Revisão Sistemática: recurso que proporciona a incorporação das evidências na prática da enfermagem. Rev. Latino-Am. Enfermagem. 2004.

GROSSECK, G.; MARINHO, S. P.; TÁRCIA, L. Educação a distância baseada na Web 2.0: a emergência de uma Pedagogia 2.0. Revista Educação e Linguagem, Vol. 12, n19, 2009.

GONÇALVES, V. M. B. A web semântica no contexto educativo. Tese (Doutorado). Universidade do Porto, 2007. Disponível em: <http://bibliotecadigital.ipb.pt/ bitstream/10198/620/1/tese_phd_vg.pdf>. Acesso em: 23 out. 2014.

HANDA, J. K.; SILVA, J. B. G. Objetos de Aprendizagem (Learning Objects). Boletim EAD - Unicamp, 31 de janeiro de 2003.2 Disponível <http://www.pucrs.br/famat/viali/tic_literatura/artigos/objetos/UNIrev_Ramos_et_al.pdf>. Acesso em: 14 abr. 2014. 


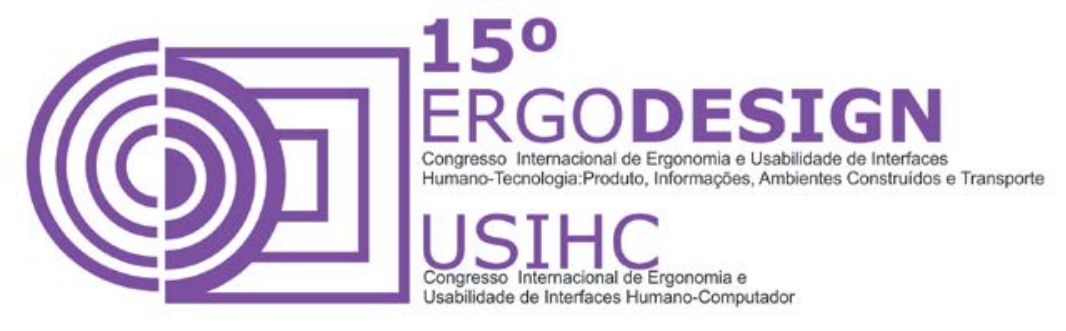

IDC, Acessado no dia 01/09/2014 disponível no site http://www.idc.com/prodserv/smartphone-os-marketshare.jsp

IBGE, Brasil em números = Brazil in figures I IBGE. Centro de Documentação e Disseminação de Informações. - Vol. 1 (1992- ). - Rio de Janeiro, 2013-

LIMA, C. R. U.; SANTAROSA, L. M. C. Acessibilidade Tecnológica e Pedagógica na Apropriação das Tecnologias de Informação e Comunicação por Pessoas com Necessidades Educacionais Especiais. In: XIV Simpósio Brasileiro de Informática na Educação - SBIE, 2003. Disponível em: <http://www.lume.ufrgs.br/bitstream/handle/10183/3709/000391527.pdf?sequence=1>. Acesso em: 1 mar. 2014.

LIMA, M. S. de; Soares, B. G. O.; Bacaltchuk, J. Psiquiatria baseada em evidências. Rev Bras Psiquiatr, set. 2000.

LINDE, K.; Willich, S. N. How objective are systematic reviews? Differences between reviews on complementary medicine. J R Soc Med. 96: 17-22, 2003.

NIELSEN, J. \& TAHIR, M., Homepage Usability: 50 Websites Deconstructed, Indianapolis: New Riders, 2002.

NIELSEN, J. \& LORANGER, H, Usabilidade na Web. Rio de Janeiro: Campus 2007

OBREGON, R. de F. A. O padrão arquetípico da alteridade e o compartilhamento de conhecimento em Ambiente Virtual de Aprendizagem. Tese (Doutorado) - UFSC, Programa de Pós-Graduação em Engenharia e Gestão do Conhecimento/EGC, 2011.

THE COCHRANE COLLABORATION (Eua) (Org.). Cochrane Handbook for Systematic Reviews of Interventions. Disponível em: <http://www.cochrane.org/training/cochrane-handbook>. Acesso em: 03 janeiro. 2014.

Torres, C. (2009). A bíblia do marketing digital. São Paulo: Novatec.

POCINHO, Margarida. Lições de metanálise. 2008. Disponível em: $<$ http://docentes.ismt.pt/ m_pocinho/Licoes_de_revisao_sistematica_e_metanalise.pdf>. Acesso em: 2014.

W3C. 2013. Disponível em: <www.w3c.org.com>. Acesso em: 2014. 